\title{
Optimal Treatment of Symptomatic Hemorrhoids
}

\author{
Seok-Gyu Song, Soung-Ho Kim \\ Department of Coloproctology, Seoul Song Do Colorectal Hospital, Seoul, Korea
}

Hemorrhoids are the most common anorectal complaint, and approximately 10 to 20 percent of patients with symptomatic hemorrhoids require surgery. Symptoms of hemorrhoids, such as painless rectal bleeding, tissue protrusion and mucous discharge, vary. The traditional therapeutic strategies of medicine include surgical, as well as non-surgical, treatment. To alleviate symptoms caused by hemorrhoids, oral treatments, such as fiber, suppositories and Sitz baths have been applied to patients. Other non-surgical treatments, such as infrared photocoagulation, injection sclerotherapy and rubber band ligation have been used to fixate the hemorrhoid's cushion. If non-surgical treatment has no effect, surgical treatments, such as a hemorrhoidectomy, procedure for prolapsed hemorrhoids, and transanal hemorrhoidal dearterialization are used.

Keywords: Hemorrhoids; Non-surgical treatment; Surgical Procedures, Operative

\section{INTRODUCTION}

Hemorrhoids are one of the most common anorectal diseases. About $5 \%$ of the total population experience hemorrhoids once in their lifetimes. In addition, roughly $50 \%$ of the individuals in their 50 's or older receive hemorrhoid treatments, and $10-20 \%$ of the individuals who receive treatment require surgery. The most common symptom of hemorrhoids is anal bleeding, and chief complaints include a perianal mass protruding from the anus and anal discharge. Most of the patients suffering from hemorrhoids have their symptoms alleviated through diet therapy, fiber supplements and medical treatments and are improved by a non-surgical therapy when necessary.

Non-surgical treatments for hemorrhoids are commonly performed for grade 1 or 2 internal hemorrhoids and are selectively performed for grade 3 hemorrhoids by using sclerotherapy, rubber band ligation and infrared coagulation. Surgical treatments include the conventional hemorrhoidectomy, the procedure for prolapsed hemorrhoid (PPH) and the transanal hemorrhoidal de-

Received: March 2, 2011 Accepted: September 2, 2011

Correspondence to: Seok-Gyu Song, M.D.

Department of Surgery, Seoul Song Do Colorectal Hospital, 366-144

Sindang 3-dong, Jung-gu, Seoul 100-453, Korea

Tel: +82-2-2250-7369, Fax: +82-2-2234-7243

E-mail: sksong00@yahoo.co.kr

(c) 2011 The Korean Society of Coloproctology

This is an open-access article distributed under the terms of the Creative Commons Attribution NonCommercial License (http://creativecommons.org/licenses/by-nc/3.0) which permits unrestricted noncommercial use, distribution, and reproduction in any medium, provided the original work is properly cited. arterialization (THD).

The purpose of treating hemorrhoids should be removing the symptoms, minimizing recurrences, and minimizing postoperative pain and complications. Further, complications, such as secondary bleeding, damage anal sphincter injury, and stenosis, which may occur after surgery, should be prevented. Recently, single or combined treatment methods for hemorrhoids, in accordance with the stage, have been introduced. In the present study, various treatment modalities for hemorrhoids are presented.

\section{ANAL DILATION}

The anal dilation procedure has been performed based on the etiology that excessive tension on the anal sphincter results in a narrowed subrectum and anal canal; then, abnormal straining for defecation generates venous congestion, which finally causes hemorrhoids. This method was introduced by Lord [1] in 1968 for the first time for grade 3 hemorrhoids, and an attempt was made to expand it to 4 finger-breath. However, this procedure was thought to produce symptoms of partial fecal incontinence due to injury of the anal sphincter in $52 \%$ of the total cases. Additionally, Konsten and Baeten [2] reported a high recurrences rate; accordingly, this procedure is currently not recommended for treating hemorrhoids.

\section{BIPOLA DIATHERMY COAGULATION}

Bipolar diathermy coagulation was introduced by Griffith et al. [3] in 1987. They reported the results of treating 43 outpatients with grade 1 or 2 hemorrhoids. According to the results, this procedure 
could be easily performed without severe pain and stenosis. This procedure is performed at the intermuscular hemorrhoid area through bipolar electrocautery using 1 -sec pulses at 20 watts with a $2.2-\mathrm{mm}$ penetration. The general success rate is about $88-100 \%$. Dennison et al. [4] reported that the results were similar to those of infrared photocoagulation, but that bipolar diathermy was more effective for simultaneously treating hemorrhoids with wide lesions. According to Yoo et al. [5], a total of 309 patients having grade 2 or 3 internal hemorrhoids were treated with bipolar diathermy, and their progressions were observed for 6 months or more, with $80 \%$ or more of the patients reporting to be satisfied with the procedure and with negligible complications other than skin tags. Yoo et al. [5] reported bipolar diathermy coagulation as a good method for treating early-stage hemorrhoids.

\section{INFRARED PHOTOCOAGULATION AND RUBBER BAND LIGATION}

Since infrared photocoagulation (IRC) was introduced by Leicester et al. [6] in 1981, this method, together with rubber band ligation, has been a mainstay procedure for treating early-stage hemorrhoids (grade 1 or 2 internal hemorrhoids). IRC works by transforming infrared radiation to heat, which generates sclerosis and fixation of hemorrhoids. The depth of penetration is $2.5 \mathrm{~mm}$, and when applied 3-4 times to the hemorrhoid area, a success rate of 67-96\% has been reported for IRC. Marques et al. [7] reported low complication rates in their comparative study with the rubber band ligation procedure. The high price of the equipment is a drawback, but selective applications will produce good results.

Rubber band ligation (RBL) was introduced by Blaisdell [8] in 1954 for the first time, and became widely known through the work of Barron [9]. RBL is the treatment of choice for early-stage hemorrhoids because it is comparatively easy to perform and can be applied repeatedly. In very few cases, postoperative septic complications have been reported, and when postoperative fever or systemic symptoms develop, the rubber band can be released to prevent those symptoms. The most common complication is pain developed within 24 hours of surgery, which can be controlled by administering pain relievers. According to a long-term follow up, recurrences outnumbered those in surgery cases $[10,11]$.

Many comparative studies on effects of RBL, IRC and sclerotherapy on early-stage hemorrhoids have been conducted. Treatment effects of RBL and IRC on grade 1 and 2 internal hemorrhoids were compared by Johanson and Rimm [12] and Templeton et al. [13]. Treatment effects were satisfactory. In case of RBL, severer anal pain was reported than in the case of IRC, but RBL showed better long-term treatment effects than IRC (IRC, 85\%; RBL, 92\%). In summary, both RBL and IRC can be used as good methods for grade 1 and 2 internal hemorrhoids. IRC can be repeatedly performed for good results if necessary, and RBL can provide good results as an office-based therapy only after the possibility of postoperative anal pain has been taken into consideration.

\section{SCLEROSING AGENT: ALUMINUM POTASSIUM SULFATE AND TANNIC ACID (ALTA)}

For relieving symptoms of early-stage hemorrhoids, an injection therapy using a sclerosing agent has been used. In most countries, $5 \%$ phenol in almond oil (PAO) has been used as a sclerosing agent for hemorrhoids. The mechanism is as follows: The agent injected into the hemorrhoid leads to an inflammatory response and as the blood flow into the hemorrhoid is interrupted, secondary fibrosis is facilitated, shrinking the hemorrhoid [14]. This method is effective for hemorrhoids with bleeding as a major symptom on a shortterm basis, but not for protruding hemorrhoids. Aluminum potassium sulfate and tannic acid (ALTA) was recently developed in Japan as an injecting sclerosing agent for treating hemorrhoids and has been actively applied to grade 2 and 3 internal hemorrhoids. This method is based on the Xiaozhiling injection introduced by Shi et al. [15] in 1970s. The ALTA sclerosing agent is administered through 4-step injections, which result in sclerosis and atrophy of the hemorrhoidal plexus, thereby treating prolapse and bleeding problems. According to a comparative study of ALTA treatments and hemorrhoidectomies for grade 3 and 4 internal hemorrhoid cases conducted by Takano et al. [16] in 2006, the one year success rates were $99 \%$ and $94 \%$, respectively, meaning that ALTA produced almost the same effects as a hemorrhoidectomy without the specific complications. Recently, a group of 338 Japanese medical institutions reported good results for ALTA. Upon injecting ALTA into grade 2,3 , and 4 hemorrhoids $(24 \%, 67 \%, 8 \%)$ of 3,519 patients, $98.1 \%$ (99.2\%, 99.8\%, 97.3\%) reported positive effects within 28 days. The recurrence rate after 2 years was $18.3 \%$, and complications included pyrexia, low blood pressure, perineal pain and rectal ulcers, but those were mild [17]. The sclerosing agent can be administered simultaneously at surgery as a combination therapy. Such a combination therapy can be selectively applied to patients with grade 1,2, and 3 hemorrhoids and to patients in high surgery-risk groups.

\section{COVENTIONAL HEMORRHOIDECTOMY}

Ligation and excision of hemorrhoids are time-tested procedures for grade 3 and 4 internal hemorrhoids. The open hemorrhoidectomy by Milligan and Morgan [18] and the closed method by Ferguson and Heater [19] are two major methods, but practically the semi-closed hemorrhoidectomy and the submucosal hemorrhoidectomy, which use the advantages of both procedures, are more commonly used. These have been the treatments of choice for various hemorrhoids and have been accepted as the "gold standard." In order to minimize damage to surrounding tissues and to maximize precise manipulation while performing the hemorrhoidectomy, new energy sources such as Ligasure have been introduced. Compared with conventional electrical cautery, this system has excellent local hemostasis effects and causes little damage to sur- 
rounding tissues due to its having only a 2-mm collateral thermal spread. Additionally, excision by using a Harmonic scalpel or Ligasure has been reported to result in less use of pain-relief agents after a hemorrhoidectomy and earlier return to work, but additional studies are required in the future for analyzing long-term hemorrhoid recurrence $[20,21]$. In an attempt to reduce pain and complications, combinations of hemorrhoidectomy and non-surgical therapies have recently been used. Because the method of the hemorrhoidectomy is selected by the surgeon, an optimal decision should be made for the benefit of the patient. Fig. 1 illustrates appropriate treatment methods according to the grade of the hemorrhoid.

\section{THROMBOSED EXTERNAL HEMORRHOID AND PROLAPSED HEMORRHOID WITH STRANGULATION}

Acute thrombosis is a main complication of external hemorrhoids. When there is engorgement of distended hemorrhoidal vessels and acute swelling of those vessels, blood may pool and consequently clot. Occasionally, the thrombus ulcerates through the skin, and the clot is extruded. This is one of the anal diseases that induces symptoms of swelling with a lump, bleeding and acute pain, which may lead to emergency medical care. Symptoms can last up to 4 weeks, and in most cases, surgical excision or drug treatments are used. Based on a recent study that reported that surgical excision was more effective for treating early symptoms than drug therapy, early surgical excision is now considered to be helpful for treating severely thrombosed external hemorrhoids [22].

Strangulated prolapsed hemorrhoids are long-history internal hemorrhoids with venous congestion, thrombus, deformation of soft tissues and edema. A part or all of the hemorrhoids is not reducible, but has strangulated out of the anal canal, resulting in necrosis or ulceration. Major methods of treatment are two: conser- vative treatment and radical treatment by emergency surgery. In case of conservative treatment, a long time is required to reduce edema and during reduction, patients experience discomfort for extended periods, so a hemorrhoidectomy is eventually required and that is the reason an emergency hemorrhoidectomy is considered. Surgeons hesitate to perform a hemorrhoidectomy due to the risk of delayed bleeding and anal stenosis, but surgical treatment can be a good choice if appropriate preservation of the mucosal wall and an adequate skin bridge are guaranteed to minimize any predictable complications $[23,24]$.

\section{PROCEDURE FOR PROLAPSED HEMORRHOIDS}

Since introduced by Longo [25], the PPH has been designed to put the hemorrhoidal plexus back into the anal canal and to fix it to preserve the anatomical and physiological functions of the anal canal, thereby avoiding the sequel of a hemorrhoidectomy. Through the PPH, excessively extended mucous membranes are removed, and internal hemorrhoids are repositioned. This is not a hemorrhoidectomy, but a hemorrhoidopexy, and eventually, internal hemorrhoids are shrunk. Table 1 presents absolute and relative contraindications for the $\mathrm{PPH}$.

Until now, more than 35 randomized studies and 7 meta-analyses on comparing the hemorrhoidectomy and the $\mathrm{PPH}$ have been re-

Table 1. Contraindications of procedure for prolapsed hemorrhoid in treatment of hemorrhoids [26]

\begin{tabular}{lc}
\hline Absolute & Relative \\
\hline Abscess/gangrene & Non-reducible prolapsed hemorrhoids \\
Full thickness rectal prolapse & Acute thrombosed internal hemorrhoids \\
Anal stenosis & Previous rectal surgery \\
\hline
\end{tabular}

Management of symptomatic hemorrhoids
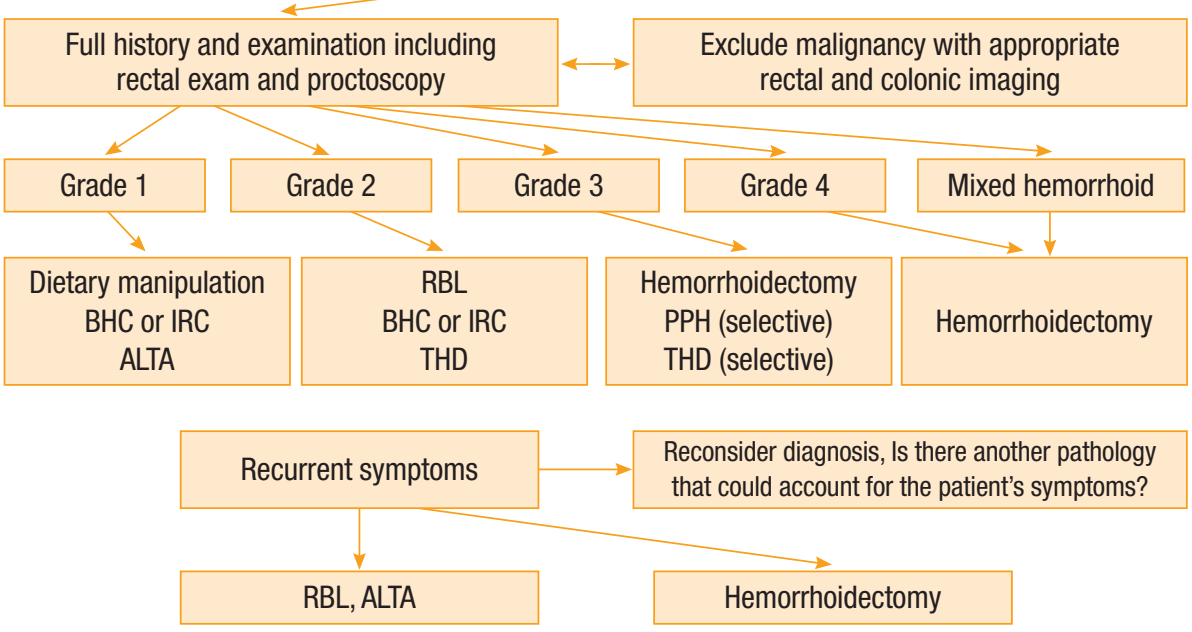

Fig. 1. Optimal treatment of symptomatic hemorrhoids. BHC, bipolar hyperthermic coagulation; IRC, infrared photocoagulation; ALTA, aluminum potassium sulfate and tannic acid; RBL, rubber band ligation; THD, transanal hemorrhoidal dearterialization; PPH, procedure for prolapsed hemorrhoid. 
ported. In terms of postoperative pain, hospital stay, and earlier return to work, the $\mathrm{PPH}$ group showed better results than the hemorrhoidectomy group. However, according to several meta-analyses, higher recurrence rates for bleeding and protrusion were reported in the PPH group, and additional surgery was required $[27,28]$. Major complications included pain and bleeding, and $0-20 \%$ of the total cases needed additional surgery due to the complications, most of which was successful [28, 29].

When hemorrhoids are wide spread over the anus, like circumferential hemorrhoids, all of the vascular plexus and deformed connective tissues that had expanded between the dentate line and the anal epithelial-cutaneous junction were theoretically excised in the past to radically remove all hemorrhoids. The method is called a Whitehead hemorrhoidectomy. However, this procedure is no longer used due to serious complications such as fecal incontinence, anal stenosis and anal mucosal eversion. In an attempt to avoid these complications, some authors tried submucosal excision of secondary hemorrhoids, the so-called modified Whitehead operation, after removing the primary hemorrhoids or tried to detach and lift mucosal flaps before excising hemorrhoidal tissues, and favorable results were reported [30]. The conventional hemorrhoidectomy is accepted as a gold standard treatment by most surgeons. However, it is important to combine appropriate numbers, widths and heights of hemorrhoids to excise by considering the flexibility of the anus to avoid excessive excision of the anal epithelial layer or mucous membranes because excessive excision of hemorrhoids to prevent recurrence may lead to complications such as anal stricture or secondary bleeding [24].

In summary, the PPH may be applied to prolapsed grade 2-3 internal hemorrhoids and to grade 4 internal hemorrhoids on a caseby-case basis. Purse-string placement is important while performing the PPH. Rates of complications, as well as fecal incontinence and continuous anal pain, are low. Compared with a hemorrhoidectomy, because the PPH offers less pain and earlier return to work, its selective use may result in good effects.

\section{TRANSANAL HEMORRHOIDAL DEARTERIALIZATION}

THD was introduced by Morinaga et al. [31] in 1995. The hemorrhoidal artery in the lower rectum is ligated under Doppler equipment to interrupt blood circulation, which contributes to shrinkage of the hemorrhoidal cushion and finally improves symptoms. Since the procedure of ligation being conducted above the dentate line to preserve the pain-sensitive anoderm was introduced in the treatment of hemorrhoids, less pain and earlier return to work have been reported compared with a hemorrhoidectomy [32]. More recently, better results have been reported by lifting the protruded mucous membranes and performing Pexis in addition to hemorrhoidal artery ligation. Moreover, comparative studies of THD, $\mathrm{PPH}$ and hemorrhoidectomy have actively been conducted. THD shows good results in terms of pain, operation time and compli- cations, and grade 2 and 3 internal hemorrhoids are considered good candidates for its use, but recurrence of symptoms is a problem in case of grade 4 internal hemorrhoids [32-35]. Rubber band ligation is recognized as the best procedure for pain and bleeding symptoms of grade 2 and 3 hemorrhoids, but the high recurrence rate is a drawback. Conaghan and Farouk [36] reported that patients who were not successful with the rubber band ligation procedure could try Doppler-guided hemorrhoid artery ligation, which might replace the conventional hemorrhoidectomy. Currently THD is recognized as a comparatively safe procedure that is certified by the US Food and Drug Administration (FDA). Moreover, THD causes less postoperative pain and is easily mastered after 3-5 times. However, further comparative studies with other hemorrhoid treatments are required for more detailed information.

\section{CONCLUSION}

If a cure based on the symptoms of hemorrhoids is to be obtained, minimally invasive treatments, such as injecting a sclerosing agent into the anal canal, rubber band ligation and recently introduced hemorrhoidal artery ligation, should be considered. In the case of recurrent and reducible hemorrhoids with severe protrusion, the $\mathrm{PPH}$ and a conventional hemorrhoidectomy may be selectively considered. Surgeons should select procedures that provide individual patients with tailored treatments based on an understanding of the indications and of the complications associated with the procedures. In conclusion, methods for treating hemorrhoids with symptoms should be selected based on the following considerations: least postoperative pain, bleeding and recurrences, and earlier return to work.

\section{CONFLICT OF INTEREST}

No potential conflict of interest relevant to this article was reported.

\section{REFERENCES}

1. Lord PH. A new regime for the treatment of haemorrhoids. Proc R Soc Med 1968;61:935-6.

2. Konsten J, Baeten CG. Hemorrhoidectomy vs. Lord's method: 17year follow-up of a prospective, randomized trial. Dis Colon Rectum 2000;43:503-6.

3. Griffith CD, Morris DL, Ellis I, Wherry DC, Hardcastle JD. Outpatient treatment of haemorrhoids with bipolar diathermy coagulation. Br J Surg 1987;74:827.

4. Dennison A, Whiston RJ, Rooney S, Chadderton RD, Wherry DC, Morris DL. A randomized comparison of infrared photocoagulation with bipolar diathermy for the outpatient treatment of hemorrhoids. Dis Colon Rectum 1990;33:32-4.

5. Yoo JJ, Lee CH, Lee KR, Part SY, Lim SW, Kim HS, et al. New technique for treatment of hemorrhoids by using bipolar hemo-coagulation (BHC). J Korean Soc Coloproctol 1997;13:229-37. 
6. Leicester RJ, Nicholls RJ, Mann CV. Infrared coagulation: a new treatment for hemorrhoids. Dis Colon Rectum 1981;24:602-5.

7. Marques CF, Nahas SC, Nahas CS, Sobrado CW Jr, Habr-Gama A, Kiss DR. Early results of the treatment of internal hemorrhoid disease by infrared coagulation and elastic banding: a prospective randomized cross-over trial. Tech Coloproctol 2006;10:312-7.

8. Blaisdell PC. Prevention of massive hemorrhage secondary to hemorrhoidectomy. Surg Gynecol Obstet 1958;106:485-8.

9. Barron J. Office ligation of internal hemorrhoids. Am J Surg 1963; 105:563-70.

10. Iyer VS, Shrier I, Gordon PH. Long-term outcome of rubber band ligation for symptomatic primary and recurrent internal hemorrhoids. Dis Colon Rectum 2004;47:1364-70.

11. Bat L, Melzer E, Koler M, Dreznick Z, Shemesh E. Complications of rubber band ligation of symptomatic internal hemorrhoids. Dis Colon Rectum 1993;36:287-90.

12. Johanson JF, Rimm A. Optimal nonsurgical treatment of hemorrhoids: a comparative analysis of infrared coagulation, rubber band ligation, and injection sclerotherapy. Am J Gastroenterol 1992;87:1600-6.

13. Templeton JL, Spence RA, Kennedy TL, Parks TG, Mackenzie G, Hanna WA. Comparison of infrared coagulation and rubber band ligation for first and second degree haemorrhoids: a randomised prospective clinical trial. Br Med J (Clin Res Ed) 1983;286:1387-9.

14. Ono T, Goto K, Takagi S, Iwasaki S, Komatsu H. Sclerosing effect of OC-108, a novel agent for hemorrhoids, is associated with granulomatous inflammation induced by aluminum. J Pharmacol Sci 2005;99:353-63.

15. Shi Z, Zhou J, He X. On treatment of third degree internal hemorrhoids with "Xiaozhiling" injection. J Trad Chin Med 1981;1: 115-20.

16. Takano M, Iwadare J, Ohba H, Takamura H, Masuda Y, Matsuo K, et al. Sclerosing therapy of internal hemorrhoids with a novel sclerosing agent: comparison with ligation and excision. Int J Colorectal Dis 2006;21:44-51.

17. Takano M, Iwadare J, Takamura H, Matsuda Y. A novel sclerosing agent for noninvasive treatments of internal hemorrhoids and prolapse. Proceedings of the Annual meeting of 2010 The American Society of Colon and Rectal Surgeons (ASCRS); 2010 May 15-9; Minneapolis, MN. p. S52-4.

18. Milligan ET, Morgan CN, Jones LE. Surgical anatomy of the anal canal and the operative treatment of haemorrhoids. Lancet 1937; 2:1119-24.

19. Ferguson JA, Heater JR. Closed hemorrhoidectomy. Dis Colon Rectum 1959;2:176-9.

20. Sakr MF. LigaSure versus Milligan-Morgan hemorrhoidectomy: a prospective randomized clinical trial. Tech Coloproctol 2010;14: 13-7.

21. Armstrong DN, Ambroze WL, Schertzer ME, Orangio GR. Harmonic Scalpel vs. electrocautery hemorrhoidectomy: a prospective evaluation. Dis Colon Rectum 2001;44:558-64.

22. Greenspon J, Williams SB, Young HA, Orkin BA. Thrombosed external hemorrhoids: outcome after conservative or surgical management. Dis Colon Rectum 2004;47:1493-8.

23. Kim JC, Hong SC. Urgent hemorrhoidectomy in the prolasped hemorrhoid with strangulation. J Korean Soc Coloproctol 1990; 6:35-40.

24. Lim SW. Analysis and measures for anal stricture following a hemorrhoidectomy. J Korean Soc Coloproctol 2006;22:293-7.

25. Longo A. Stapled anopexy and stapled hemorrhoidectomy: two opposite concepts and procedures. Dis Colon Rectum 2002;45: 571-2.

26. Corman ML, Gravie JF, Hager T, Loudon MA, Mascagni D, Nystrom PO, et al. Stapled haemorrhoidopexy: a consensus position paper by an international working party - indications, contra-indications and technique. Colorectal Dis 2003;5:304-10.

27. Shao WJ, Li GC, Zhang ZH, Yang BL, Sun GD, Chen YQ. Systematic review and meta-analysis of randomized controlled trials comparing stapled haemorrhoidopexy with conventional haemorrhoidectomy. Br J Surg 2008;95:147-60.

28. Tjandra JJ, Chan MK. Systematic review on the procedure for prolapse and hemorrhoids (stapled hemorrhoidopexy). Dis Colon Rectum 2007;50:878-92.

29. Senagore AJ, Singer M, Abcarian H, Fleshman J, Corman M, Wexner $\mathrm{S}$, et al. A prospective, randomized, controlled multicenter trial comparing stapled hemorrhoidopexy and Ferguson hemorrhoidectomy: perioperative and one-year results. Dis Colon Rectum 2004;47:1824-36.

30. Kim JH, Kang DW, Sun BH. Revisit the original whitehead hemorrhoidectomy: the postoperative results of W-shaped circular incision \& preservation of perianal skin. J Korean Soc Coloproctol 1998;14:101-7.

31. Morinaga K, Hasuda K, Ikeda T. A novel therapy for internal hemorrhoids: ligation of the hemorrhoidal artery with a newly devised instrument (Moricorn) in conjunction with a Doppler flowmeter. Am J Gastroenterol 1995;90:610-3.

32. Giordano P, Overton J, Madeddu F, Zaman S, Gravante G. Transanal hemorrhoidal dearterialization: a systematic review. Dis Colon Rectum 2009;52:1665-71.

33. Festen S, van Hoogstraten MJ, van Geloven AA, Gerhards MF. Treatment of grade III and IV haemorrhoidal disease with PPH or THD: a randomized trial on postoperative complications and short-term results. Int J Colorectal Dis 2009;24:1401-5.

34. Ratto C, Donisi L, Parello A, Litta F, Doglietto GB. Evaluation of transanal hemorrhoidal dearterialization as a minimally invasive therapeutic approach to hemorrhoids. Dis Colon Rectum 2010;53: 803-11.

35. Sakr MF, Moussa MM. LigaSure hemorrhoidectomy versus stapled Hemorrhoidopexy: a prospective, randomized clinical trial. Dis Colon Rectum 2010;53:1161-7.

36. Conaghan P, Farouk R. Doppler-guided hemorrhoid artery ligation reduces the need for conventional hemorrhoid surgery in patients who fail rubber band ligation treatment. Dis Colon Rectum 2009;52:127-30. 